\title{
Recent results from the OPERA experiment
}

\author{
F. Di CApua on behalf of the OPERA Collaboration \\ INFN, Sezione di Napoli - Napoli, Italy
}

(ricevuto il 10 Novembre 2009; pubblicato online il 18 Gennaio 2010)

\begin{abstract}
Summary. - Several experiments carried out in the last decades with atmospheric and accelerator neutrinos, as well as with solar and reactor neutrinos, contributed to our present understanding of neutrino mixing (see, e.g., Strumia and Vissani [arXiv:hep-ph/0606054] for a review). The OPERA experiment aims at measuring the first detection of neutrino oscillation in appearance mode through the detection of $\nu_{\tau}$ in an almost pure $\nu_{\mu}$ beam produced at CERN SPS (CNGS), $730 \mathrm{~km}$ far from the detector. The $\nu_{\tau}$ appearance signal is detected through the measurement of the decay daughter particles of the $\tau$ lepton produced in CC $\nu_{\tau}$ interactions. Since the short-lived $\tau$ particle has, at the energy of the beam, an average decay length of about $\sim 1 \mathrm{~mm}$, a micrometric detection resolution is needed. Runs with CNGS neutrinos were succesfully carried out in 2007 and 2008 with the detector fully operational with its related facilities for the emulsion handling and analysis. After a brief description of the beam and of the experimental setup we report on the collection, reconstruction and analysis procedures of first samples of neutrino interaction events.
\end{abstract}

PACS 13.15.+g - Neutrino interactions.

PACS 14.60.Lm - Ordinary neutrinos $\left(\nu_{\mathrm{e}}, \nu_{\mu}, \nu_{\tau}\right)$.

PACS 29.40.Rg - Nuclear emulsions.

\section{1. - Introduction}

Evidence for neutrino oscillations have been unambiguously observed by several experiments [1], by using atmospheric and accelerator neutrinos, as well as with solar and reactor neutrinos. However, as far as the atmospheric neutrino sector is concerned, despite the fact that there are many experimental indications supporting the $\nu_{\mu} \rightarrow \nu_{\tau}$ solution, a direct evidence of the $\nu_{\tau}$ appearance is still missing.

OPERA [2] uses the long-baseline $(L=730 \mathrm{~km})$ CNGS neutrino beam [3] from CERN to LNGS. The beam is designed to provide $4.5 \cdot 10^{19}$ proton-on-target/year (p.o.t./y) with a running time of 200 days per year. The beam parameters have been optimized in order to maximize the number of $\nu_{\tau}$ charged current interactions in the OPERA detector. The average neutrino energy is $\langle E\rangle=17 \mathrm{GeV}$ with a small contamination of $\bar{\nu}_{\mu}(4.0 \%)$ and of $\nu_{e}, \bar{\nu}_{e}$ (less than $\left.1 \%\right)$. 


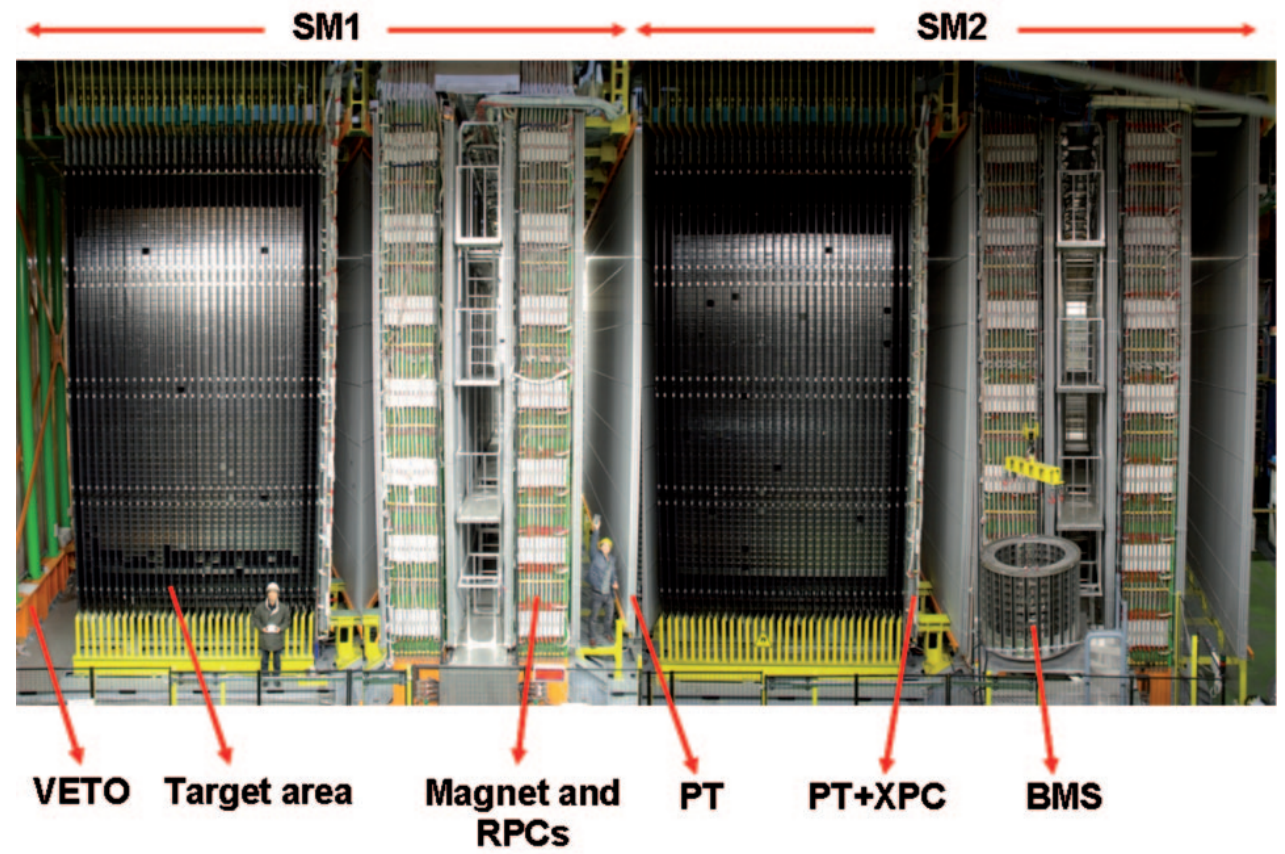

Fig. 1. - View of the OPERA detector; the neutrino beam enters from the left. The upper horizontal lines indicate the position of the two identical supermodules (SM1 and SM2). The "target area" is made of walls filled with ECC bricks interleaved with planes of plastic scintillators (TT). Arrows show the position of the VETO planes, the drift tubes (PT) pulled alongside the XPC, the magnets and the RPC installed between the magnet iron slabs. The Brick Manipulator System (BMS) is also visible. See [2] for more details.

In 5 years of data taking, OPERA is able to observe 10 to $15 \nu_{\tau}$ events after oscillation at full mixing in the range $2.5 \times 10^{-3}<\Delta m^{2}<3 \times 10^{-3} \mathrm{eV}^{2}$, with a total background of 0.75 events.

\section{2. - The OPERA detector}

The detection of the short-lived $\tau$ lepton $(\mathrm{c} \tau=87.11 \mu \mathrm{m})$ produced in the chargedcurrent (CC) interaction of a $\nu_{\tau}$ is mandatory. This sets two conflicting requirements: a large target mass to collect enough statistics and an extremely high spatial accuracy to observe the short-lived $\tau$ lepton.

The $\tau$ is identified by the detection of its characteristic decay topologies either in one prong (electron, muon or hadron) or in three-prongs; its short track is measured with a large mass target made of $1 \mathrm{~mm}$ thick lead plates (target mass and absorber material) interspaced with thin nuclear emulsion films (high-accuracy tracking devices). This detector is historically called Emulsion Cloud Chamber (ECC).

The OPERA apparatus (fig. 1) consists of 2 identical parts called super-modules (SMs). Each super-module consists of a target section of about 625 tons made of emulsion/lead bricks, of a scintillator strips tracker detector (TT) and of a muon spectrometer. TT planes serve as trigger devices and allow selecting the brick containing a neutrino 


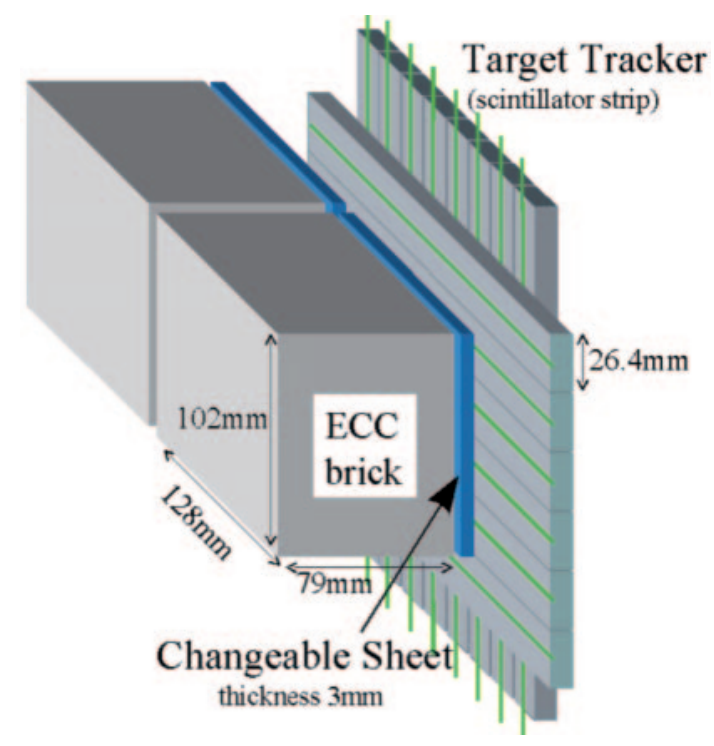

Fig. 2. - Schematic view of two bricks with their Changeable Sheets and target tracker planes.

interaction. The muon spectrometer at the downstream end of each SM, allows measuring charge and momentum of penetrating tracks. A large size anti-coincidence detector (VETO), made of two glass RPC planes mounted in front of the first target, allows to reject charged particles originating from outside the target fiducial region coming from neutrino interactions in the surrounding rock material.

Each target section is a sequence of vertical steel containers (walls) hosting the bricks, interleaved with double-layered plastic scintillator strips planes as Target Trackers (TT). Each brick wall contains $\sim 2900$ bricks for a total of 150000 bricks in the whole apparatus.

A target brick consists of 56 lead plates of $1 \mathrm{~mm}$ thickness interleaved with 57 emulsion films [4]. The transverse dimensions of a brick are $12.8 \times 10.2 \mathrm{~cm}^{2}$ and the thickness along the beam $\mathrm{z}$ direction is $7.9 \mathrm{~cm}$ (about 10 radiation lengths). The weight is $8.3 \mathrm{~kg}$.

An OPERA film has 2 emulsion layers (each $44 \mu \mathrm{m}$ thick) on both sides of a transparent triacetylcellulose base $(205 \mu \mathrm{m}$ thick). The total thickness is $293 \pm 5 \mu \mathrm{m}$. The large amount of emulsion films used, translated in surface, covers $100,000 \mathrm{~m}^{2}$.

In order to reduce the emulsion scanning load the use of Changeable Sheets (CS) film interfaces [5], successfully applied in the CHORUS experiment [6], was extended to OPERA. Tightly packed doublets of emulsion films are glued to the downstream face of each brick and can be removed without opening the brick. The global layout of brick, CS and TT is schematically shown in fig. 2 .

The scanning of emulsion films is performed with two different types of automatic microscopes: the European Scanning System (ESS) [7,8] and the Japanese S-UTS [9]. Microscope systems have comparable performances ensuring a scanning speed two order of magnitude greater than that of the systems used in past experiments, and a spatial and angular resolution of the order of $\sim 1 \mu \mathrm{m}$ and $1 \mathrm{mrad}$, respectively. 


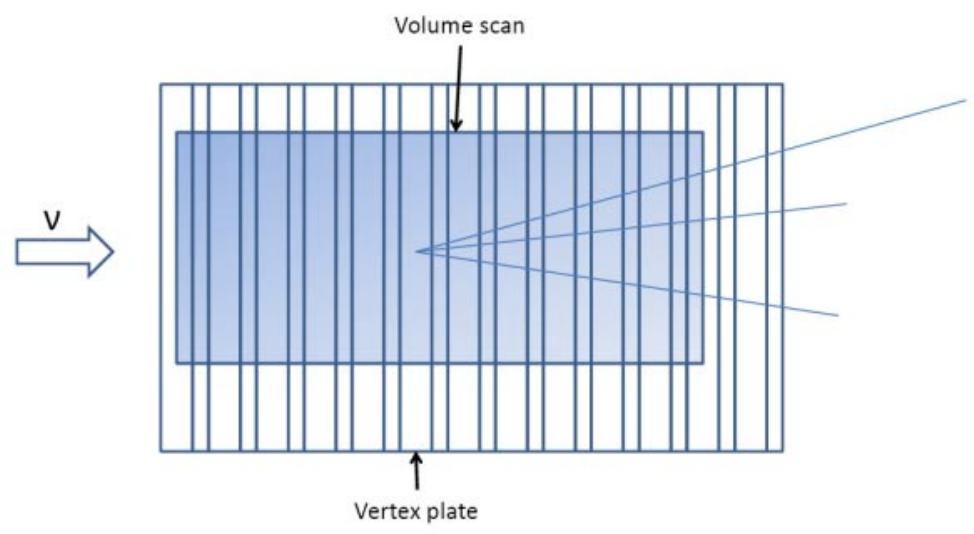

Fig. 3. - Schematic view of the volume scan performed around the stopping point of the track.

\section{3. - Event analysis chain}

We describe in the following the breakdown of the different steps carried out to analyze neutrino interaction events from the identification of the "fired" brick up to the detailed kinematical analysis of the vertex in the emulsion films.

Once a trigger in the electronic detectors is selected to be compatible with an interaction inside a brick, electronic detector data are processed by a software reconstruction program that selects the brick with the highest probability to contain the neutrino interaction vertex. This brick is removed from the target wall by the BMS and exposed to $\mathrm{X}$-rays for film-to-film alignment. There are two independent X-ray exposures: the first one ensures a common reference system to the CS film doublet and the most downstream film of the brick (frontal exposure); the second one produces thick lateral marks on the brick edges, used for film to film alignment inside the brick.

After the first X-ray exposure the CS doublet is detached from the brick and developed underground, while the brick is kept in a box made of $5 \mathrm{~cm}$ thick iron shielding to reduce the radioactivity background. The scanning of the CS is done in two laboratories hosting a farm of microscopes (Scanning Stations), one at LNGS and the other in Nagoya. CS films are analyzed with a procedure called "general scanning" that looks for tracks in all the available angular range (typically $\pm 400 \mathrm{mrad}$ around the perpendicular to the film) in an area of about $50 \mathrm{~cm}^{2}$ around the TT prediction. The residuals with respect to the TT prediction, are of the order of about $1 \mathrm{~cm}$ in position and $20 \mathrm{mrad}$ in angle.

If the CS scanning detects tracks compatible with those reconstructed in the electronic detectors the second X-ray exposure (lateral marking) is performed and the brick is brought to the surface laboratory. It is exposed to cosmic rays for about 24 hours in a dedicated pit in order to select high-energy cosmic muons to provide straight tracks for a sub-micrometric film-to-film alignment. The brick emulsion films are then developed and dispatched to the various scanning laboratories in Europe and Japan.

All tracks measured in the CS are sought in the most downstream films of the brick and followed back until they are not found in three consecutive films. The stopping point is considered as the signature either for a primary or a secondary vertex. The existence of the vertex is then confirmed by scanning a volume with a transverse size of $1 \mathrm{~cm}^{2}$ for 11 films in total, upstream and downstream of the stopping point (see fig. 3). 

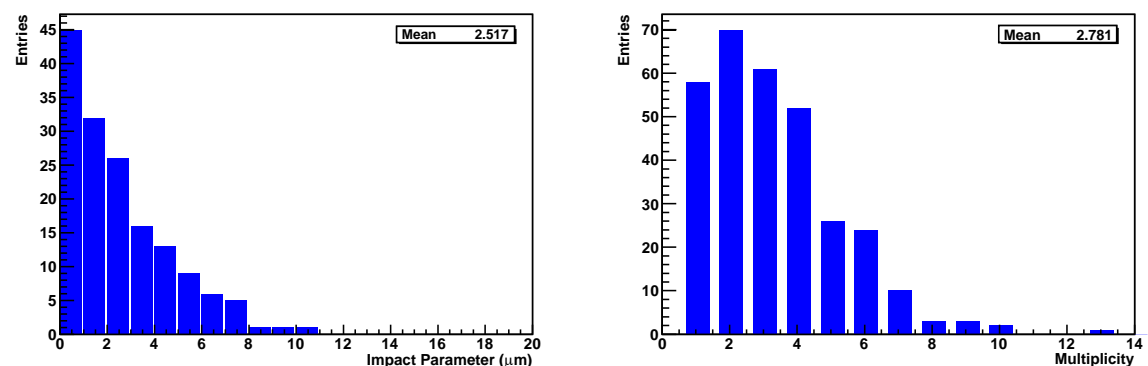

Fig. 4. - Left panel: impact parameter distribution of the muon track in CC events with respect to the reconstructed vertices. Right panel: charged track multiplicity distribution of the events.

The track impact parameter distribution of the muon in CC events with respect to the reconstructed vertex position and the event track multiplicity distribution are shown in fig. 4. As expected, the impact parameter distribution is peaked at zero and has a mean value of $2.5 \mu \mathrm{m}$. The multiplicity distribution shows the anticipated enhancements for even track numbers due to the preferred interaction of neutrinos with neutrons.

\section{4. - First results and decay topologies}

After a short commissioning run in 2006 the CNGS operation started on September 2007 at rather low intensity. The first event inside the OPERA target was observed on October 3rd. Unfortunately, due to a fault of the CNGS facility, the physics run lasted only a few days. During this run $8.2 \times 10^{17}$ protons on target (p.o.t.) were accumulated: this corresponds to about $\sim 3.6$ effective nominal days of running. With such an integrated intensity 32 neutrino interactions in the bricks and 3 in the scintillator material of the target tracker were expected; we actually observed 38 events on time with the arrival of the beam at Gran Sasso.

A much longer run took place in 2008 when $1.782 \times 10^{19}$ protons were delivered on the CNGS target with a mean value of $\sim 2 \times 10^{13}$ protons per extraction. OPERA collected 10100 events on time and among them about 1700 interactions in the target region. At the time of the conference 315 neutrino interactions were located and their topology was fully reconstructed: among them 270 were CC events while 45 were NC events.

Among these events secondary decay topologies are expected, mainly due to charm production. The detection of these events have a great importance in OPERA. Since charm decays exhibit the same topology as $\tau$ decays, measuring the charm-like event reconstruction efficiency provides an important cross-check of the $\tau$ event reconstruction capability. On the other hand, charm events are a potential source of background, in particular if the muon at the primary vertex is not identified (see fig. 5). Therefore, searching for charm-decays in events with the primary muon correctly identified provides a direct measurement of this background.

The event in fig. 6 has high track multiplicity at the primary vertex and one of the scan-back track shows a kink topology. The measured decay angle is $204 \mathrm{mrad}$ and the flight length of the decaying particle is $3247 \mu \mathrm{m}$. The decay occurred in the third lead plate downstream of the interaction plate. No large angle tracks are produced at the decay vertex. The daughter momentum, measured by using the Multiple Coulomb 

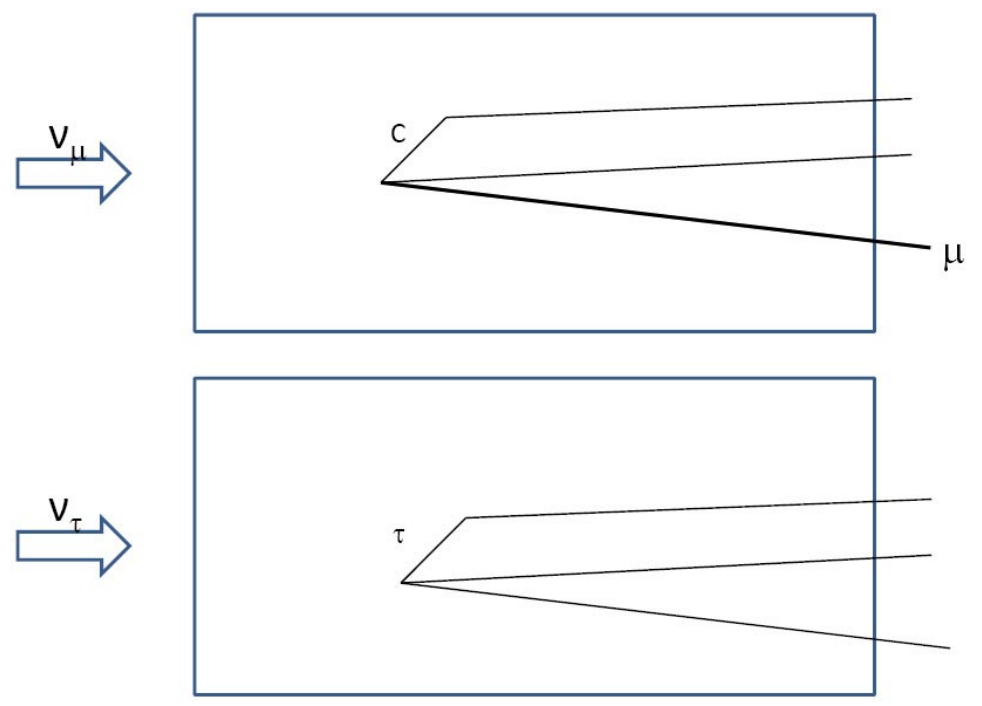

Fig. 5. - Schematic view of the charm and tau decay topologies.
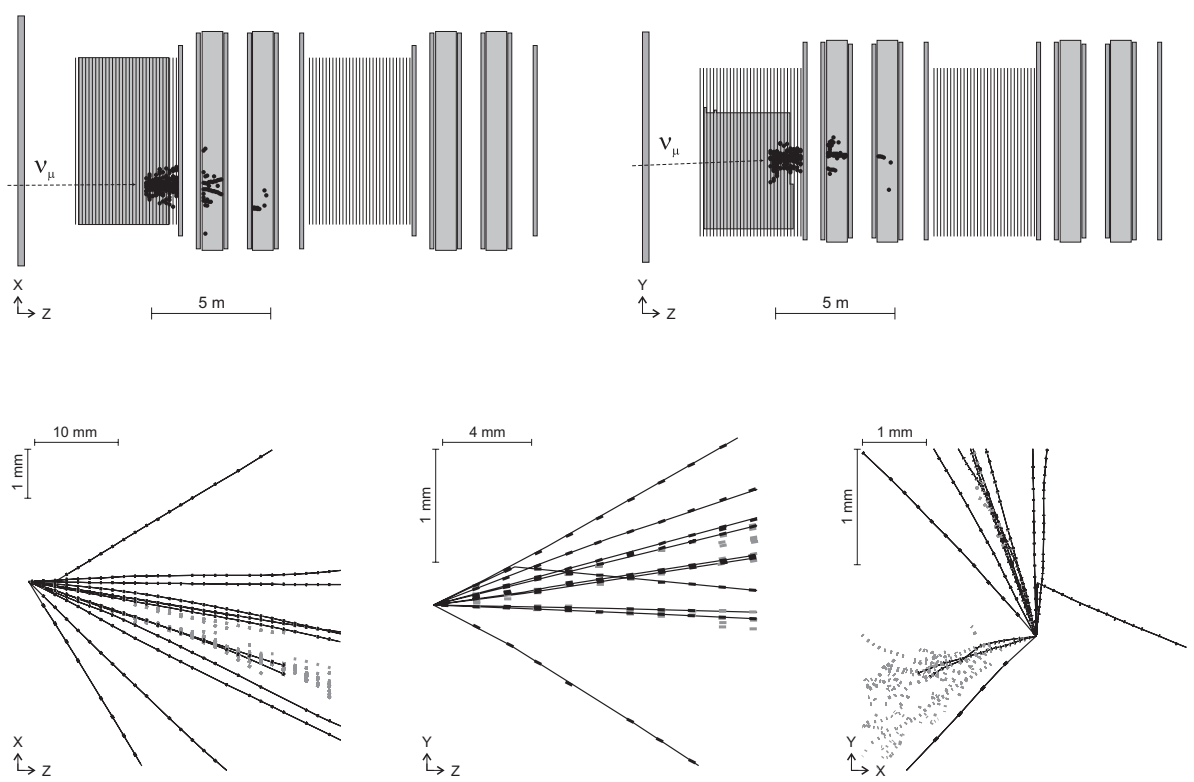

Fig. 6. - Online display of the OPERA electronic detector of a $\nu_{\mu}$ charged-current interaction with a charm-like topology (top panel). The emulsion reconstruction is shown in the bottom panels where the charm-like topology is seen as a track with a kink: top view (bottom left), side view (bottom center), frontal view (bottom right). The dots visible in the lower panel are due to an electromagnetic shower. 

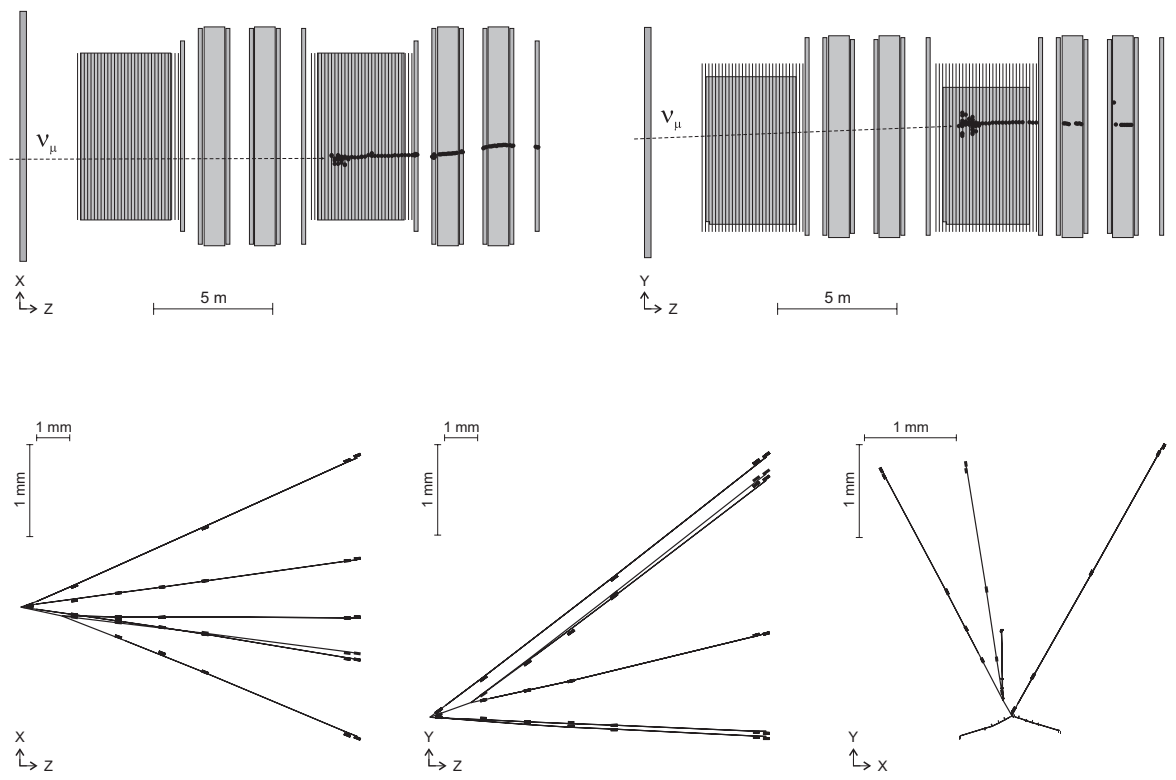

Fig. 7. - Online display of the OPERA electronic detector of a $\nu_{\mu}$ charged-current interaction with a charm-like topology (top panel). The emulsion reconstruction is shown in the bottom panels where the charm-like topology is seen as a three-prongs secondary vertex: top view (bottom left), side view (bottom center), frontal view (bottom right).

Scattering technique [10] is $3.9_{-0.9}^{+1.7} \mathrm{GeV} / c$ at the $90 \%$ CL. Therefore, at the $90 \%$ CL the transverse momentum ranges between $610 \mathrm{MeV} / c$ and $1140 \mathrm{MeV} / c$. The daughter particle being a hadron, we computed the probability that a hadron interaction mimics a hadronic charm decay. According to the FLUKA Monte Carlo [11] the probability that a hadron interaction mimics a charm-decay with transverse momentum larger than $610 \mathrm{MeV} / c$ is only $4 \times 10^{-4}$.

A second charm-like topology is shown in fig. 7. A 4-prong primary vertex is observed originating at a depth of about $30 \mu \mathrm{m}$ in the upstream lead plate. The charmed hadron track points to a 3-prong decay vertex located at a distance of $1150 \mu \mathrm{m}$ from the primary vertex (200 $\mu \mathrm{m}$ inside the lead). The interaction occurs downstream in the brick and the tracks only cross four emulsion films and the CS doublet (the two most downstream hits in the figure). The muon track and the charm candidate track lie in a back-to-back configuration $\left(\Delta \phi \simeq 150^{\circ}\right)$. The momenta of the daughter tracks have also been measured by extracting the downstream brick and using the Multiple Coulomb Scattering technique. The measured values are $p_{1}=2.4_{-0.6}^{+1.3}, p_{2}=1.3_{-0.3}^{+0.4}$ and $p_{3}=1.2_{-0.4}^{+1.7} \mathrm{GeV} / \mathrm{c}$ (transverse momenta of about 610,90 and $340 \mathrm{MeV} / c$, total momentum: $4.8_{-0.8}^{+2.2} \mathrm{GeV} / c$ ), at the $90 \%$ CL. The probability of a decay in flight of a $K$ is about $10^{-3}$. The probability of a hadron interaction has been evaluated using FLUKA and amounts to $10^{-6}$. Assuming a $D \rightarrow K \pi \pi$ decay, an invariant mass of $1.1_{-0.1}^{+0.2} \mathrm{GeV} / c^{2}$ is obtained. On the other hand, assuming a $D_{s} \rightarrow K K \pi$ decay an invariant mass of $1.5_{-0.1}^{+0.4} \mathrm{GeV} / c^{2}$ is derived. In the latter case the invariant mass is consistent with the mass of a charmed hadron while in the first case the consistency is marginal. 


\section{5. - Conclusions and outlook}

In the 2008 run OPERA detected about 1700 neutrinos interacting in the targets, consistently with the value expected from the CNGS integrated intensity. At the time of the La Thuille Conference 315 interactions were located in the bricks: $45 \mathrm{NC}$ and $270 \mathrm{CC}$ interactions. The Scanning Stations and the scanning laboratories of the Collaboration are completing the analysis of the bricks.

A new physics run was started in June 2009 and completed in November 2009: a total integrated intensity of $3.5 \times 10^{19}$ protons on target has been delivered, 3700 neutrino interactions in the target have been collected. With such statistics we expect to observe the first $\tau$ event candidates.

\section{REFERENCES}

[1] Strumia A. and Vissani F., Neutrino masses and mixings and. ., arXiv:hep-ph/0606054.

[2] Acquafredda R. et al. (OPERA Collaboration), JINST, 4 (2009) P04018.

[3] CNGS project: http://proj-cngs.web.cern.ch/proj-cngs/.

[4] Nakamura T. et al., Nucl. Instrum. Methods A, 556 (2006) 80.

[5] Anokhina A. et al. (OPERA Collaboration), JiNST, 3 (2008) P07005.

[6] Eskut E. et al. (CHORUS Collaboration), Nucl. Instrum. Methods A, 401 (1997) 7.

[7] Rosa G. et al., Nucl. Instrum. Methods A, 394 (1997) 357; Armenise N. et al., Nucl. Instrum. Methods A, 551 (2005) 261; De SERIO M. et al., Nucl. Instrum. Methods A, 554 (2005) 247; Arrabito L. et al., Nucl. Instrum. Methods A, 568 (2006) 578; Kreslo I. et al., JINST, 3 (2008) P04006.

[8] Arrabito L. et al., JiNST, 2 (2007) P05004.

[9] Aoki S. et al., Nucl. Instrum. Methods B, 51 (1990) 466; NAKAno T., PhD Thesis, University of Nagoya (1997); NAKANO T. (CHORUS COllaboration), International Europhysics Conference on High-Energy Physics (HEP 2001), Budapest, Hungary, 12-18 July 2001.

[10] De Serio M. et al., Nucl. Instrum. Methods A, 512 (2003) 539; Kodama K. et al., Nucl. Instrum. Methods A, 574 (2007) 192.

[11] For details on the FLUKA Monte Carlo we refer to http://www.fluka.org. 